\title{
METHODENLEHRE DER SOZIALWISSENSCHAFTEN
}

VON

\section{FELIX KAUFMANN}

DR. JUR. ET PHIL., PRIVATDOZENT AN DER UNIVERSTTÄT WIEN

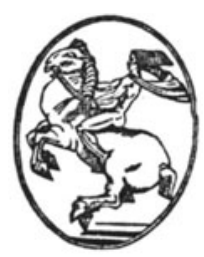

WIEN

VERLAG VON JULIUS SPRINGER

1936 
ISBN 978-3-7091-5967-5 ISBN 978-3-7091-6001-5 (eBook) DOI 10.1007/978-3-7091-6001-5

ALLE RECHTE, INSBESONDERE DAS DER UBERSETZUNG IN FREMDE SPRACHEN, VORBEHALTEN

COPYRIGHT 1936 BY JULIUS SPRINGER IN VIENNA 


\section{Vorwort.}

Der Plan dieser Arbeit ist der Überzeugung entsprungen, daß viele der heftigsten methodologischen Kontroversen innerhalb der Sozialwissenschaften zur Entscheidung reif sind. Er liegt mehr als 15 Jahre zurück; aber für die Durchführung bedurfte es sorgfältiger Vorbereitungen, über deren Art meine in den Anmerkungen zitierten Publikationen teilweise Aufschluß geben. Neben dem Erkenntnisziel jene UUberzeugung zu begründen hat mir das didaktische Ziel vorgeschwebt, dem mit abstrakteren Problemen befaßten Sozialforscher und auch dem vorgeschrittenen Studenten einen Einblick in die Verflechtung der gesellschaftstheoretischen Probleme mit solchen der allgemeinen Erkenntnistheorie und der Theorie scheinbar weit abseits liegender Wissenschaften zu geben und ineins damit - eine Reihe eingewurzelter Vorurteile auszumerzen, die dem Verständnis des Sinns sozialwissenschaftlicher Forschung und ihrer ungestörten Entwicklung im Wege stehen.

Die Orientierung an diesen Zielen führt zur Konzentration auf die grundsätzlich bedeutsamen wissenschaftstheoretischen Zusammenhänge und zu fast völliger Ausschaltung der gegenüber diesem Untersuchungszentrum peripheren Betrachtungen, mögen diese auch dogmengeschichtlich, erkenntnispsychologisch oder wissenssoziologisch noch so interessant sein. Demgemäß ist alle Kritik in diesem Buche Prinzipienkritik.

Diese Feststellung scheint eine ergänzende Angabe darüber zu erfordern, von welchem philosophischen "Standpunkt" her die Prinzipienkritik erfolgt. Aber dies trifft nicht zu; der gegensätzliche Anschein entsteht durch eine im folgenden näher zu kennzeichnende Fehlauffassung des philosophischen Denkens. Auch möchte ich bemerken, daß meine Arbeit nicht als phänomenologische Theorie der Sozialwissenschaften zu bezeichnen ist, obwohl die Werke des Begründers der transzendentalen Phänomenologie, des großen Philosophen Edmund Husserl, meine Gedanken stark und nachhaltig beeinflußt haben. Denn die in diesem Buche durchgeführten methodologischen Analysen stehen noch diesseits der 
Problemstellung der transzendentalen Phänomenologie; ihr Ziel ist „Formalkritik“, nicht „Transzendentalkritik“, wenn man diese beiden Begriffe im Sinne HusserLs vensteht.

Auch in den Rahmen einer bestehenden gesellschaftstheoretischen, wirtschaftstheoretischen oder rechtstheoretischen Schule läßt sich die Arbeit nicht zwanglos einordnen; doch darf nicht unerwähnt bleiben, daß ich mich besonders zwei Sozialforschern für eine Fülle von Anregungen, die ich aus ihren Schriften und aus langjührigen Diskussionen schöpfen konnte, verpflichtet weiß: Hans Kelsen und Ludwig Mises.

Die Gründe, die für den Aufbau bestimmend waren, sind der folgenden Einleitung zu entnehmen. An dieser Stelle will ich nur darauf hinweisen, daß die Anwendungen auf sozialtheoretische Probleme, die im zweiten Teil von den allgemein wissenschaftstheoretischen Besinnungsergebnissen des ersten Teils gemacht werden, vielfältig vermehrt werden können - was hoffentlich bald durch Zusammenarbeit einer größeren Anzahl von Forschern geschehen wird - und daß ich darum nicht allzu viel Gewicht auf eine durchwegs bis ins einzelne gehende Korrespondenz der Analysen in den beiden Teilen der Arbeit gelegt habe. Eine solche hätte die mir weit wesentlicher erscheinende Geradlinigkeit der Darstellung der allgemein wissenschaftstheoretischen Problematik beeinträchtigt.

Die in den Anmerkungen verzeichnete Literatur ist bloß ein kleiner Bruchteil des gedanklich verarbeiteten Schrifttums. Ich habe mich meist darauf beschränkt, diejenigen Werke anzuführen, auf welche im Text ausdrücklich Bezug genommen wird.

Für kritische Durchsicht des Manuskripts und der Druckbogen möchte ich einer Reihe von Freunden in verschiedenen Ländern, insbesondere Dr. KarL BoDE, dzt. St. Johns College, Cambridge und Dr. Alfred Schütz, Wien, auf das herzlichste danken. Dr. BodE hat auch die große Mühe der Herstellung der beiden Register auf sich genommen.

Wien, im November 1935.

F. KAUFMANN. 


\section{Inhaltsverzeichnis.}

Einleitung: Seite

Einleitung: Zur Problemstellung und Gliederung der Arbeit ..... 1

Erster Teil. Elemente der allgemeinen Wirtschaftstheorie.

1. Philosophische Grundüberlegungen $\ldots \ldots \ldots \ldots \ldots \ldots \ldots \ldots \ldots$

2. Das logisch-mathematische Denken $\ldots \ldots \ldots \ldots \ldots \ldots \ldots \ldots \ldots$

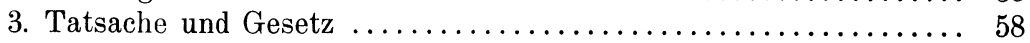

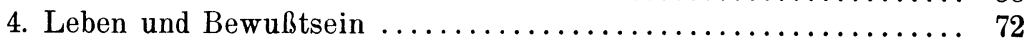

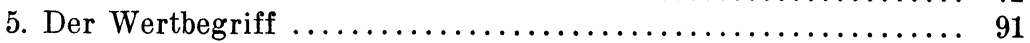

6. Wissenschaftstheorie und Metaphysik ................... 110

7. Entwurf eines methodologischen Universalschemas ......... 121

$\mathrm{Z}$ weiter Teil. Der Methodenstreit in den Sozialwissenschaften.

Vorbereitende Bemerkungen $\ldots \ldots \ldots \ldots \ldots \ldots \ldots \ldots \ldots \ldots \ldots . \ldots 129$

1. Sozialwissenschaften und Naturwissenschaften $\ldots \ldots \ldots \ldots \ldots 131$

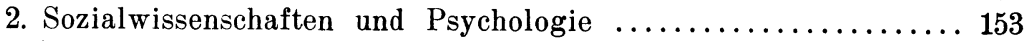

3. Das Wertproblem in den Sozialwissenschaften $\ldots \ldots \ldots \ldots \ldots 169$

4. Das „Historische“ in den Sozialwissenschaften ............. 193

5. Sozialwissenschaftliche Grundbgeriffe ................. 204

6. Sozialgesetze und Idealtypen ...................... 218

7. Der Weg zur Utberwindung des Methodenstreites ........... 235

8. Bemerkungen zum Methodenstreit um die Grenznutzentheorie.. 255

9. Der Begriff des positiven Rechts und die Reine Rechtslehre ... 291

Anmerkungen: Zusätze und Literaturhinweise ............... 311

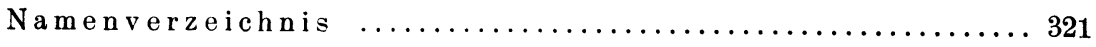

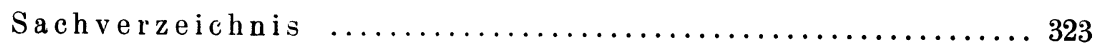

